\title{
Stable DFT Codes and Frames
}

\author{
Paulo J. S. G. Ferreira, Member, IEEE and José M. N. Vieira, Member, IEEE
}

\begin{abstract}
Discrete Fourier transform codes (DFT codes) or real number codes have been studied and recognized as useful (as joint source-channel codes, for example) but are not stable under bursty losses. This letter introduces a two-channel DFT code with an interleaver and shows that its numerical stability far exceeds that of the corresponding single-channel DFT code (the ratio of the frame bounds for the two-channel system can be smaller by many orders of magnitude). This leads to a stable way of dealing with bursts of errors using DFT codes.
\end{abstract}

Index Terms-Concatenated codes, condition number, eigenvalues, error control coding, frame bounds, frames, interpolation, numerical stability, real codes.

\section{INTRODUCTION}

$\mathbf{T}$ HE BASIC building block in discrete Fourier transform (DFT) codes is depicted in Fig. 1(a). The original data vector $a$, with $K$ samples, is transformed, and then zero-padded to obtain a total of $N$ samples. The inverse DFT (IDFT) of this $N$-dimensional vector is the coded vector $x$. The first transform is optional, since the transformed data $b$ can be regarded as the original data.

The connection between error control coding and the transform methods of signal processing is known [1], [2]. The problem can be discussed in terms of frames [3], showing the equivalence (for this particular purpose) of the frame algorithm, Projections onto Convex Sets, and the Gerchberg-Papoulis algorithm. Some problems in interpolation, spectrum analysis, error control coding, and fault-tolerant computing can also be related [4]. It is possible to deal with errors, rather than erasures, in signals and images [5], [6]. Quantized frames [7], estimates for the frame bounds [8], and sampling with unknown locations [9] have also been discussed.

Previous work on erasure correction using DFT codes [10] yielded upper and lower error bounds as a function of the number of iterations, the signals for which the bounds are attained, the erasure patterns for which the convergence rate of the frame algorithm is maximum or minimum, and more. The convergence rate in the lowpass case is minimum for contiguous erasures [10], in which case the problem can be extremely ill-posed. For more on bursty erasures, see [11] and [12]. Extrapolation and superresolution are problems made difficult (or interesting) by the contiguous distribution of the unknown segments of the data.

Manuscript received June 11, 2002; revised August 20, 2002. This work was partially supported by the Fundacão para a Ciéncia e Tecnologia. The associate editor coordinating the review of this manuscript and approving it for publication was Prof. Soo-Chang Pei.

The authors are with the Departamento Electrónica e Telecomunicações/IEETA Universidade de Aveiro, 3810-193 Aveiro, Portugal (e-mail: pjf@det.ua.pt; vieira@det.ua.pt).

Digital Object Identifier 10.1109/LSP.2002.807876

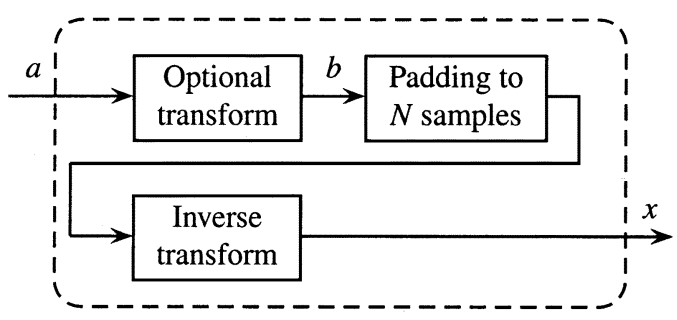

(a)

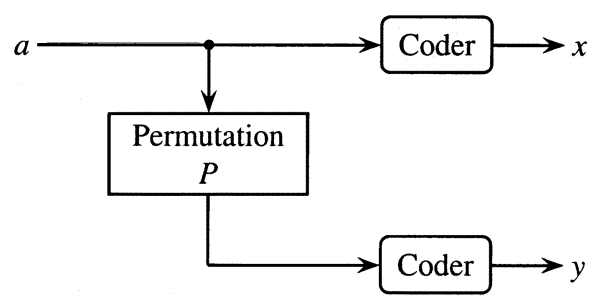

(b)

Fig. 1. (a) Single-channel coder. (b) Two-channel coder. To obtain the same redundancy or code rate in both cases, take $a \in \mathbb{R}^{K}$ and $x \in \mathbb{R}^{2 N}$ in (a) and $a \in \mathbb{R}^{K}, x \in \mathbb{R}^{N}, y \in \mathbb{R}^{N}$ in (b).

This work introduces and studies a new DFT code [Fig. 1(b)] capable of handling error bursts without numerical problems. A structure less sensitive to variations in the error pattern cannot be obtained simply by interleaving the coded data, because there are patterns that would be mapped by the interleaver into contiguous patterns. Two data paths, one in which the data are coded normally, and another in which they are interleaved, as in Fig. 1(b), lead to more uniform performance with respect to error patterns.

To compensate for the existence of two data paths and obtain one-channel and two-channel coders with the same overall degree of redundancy (or code rate), simply take $a \in \mathbb{R}^{K}$ and $x \in \mathbb{R}^{2 N}$ in the one-channel case [Fig. 1(a)] and $a \in \mathbb{R}^{K}$, $x \in \mathbb{R}^{N}, y \in \mathbb{R}^{N}$ in the two-channel case [Fig. 1(b)].

Another motivation for studying the system of Fig. 1(b) concerns the performance of finite-field concatenated codes with an interleaver, discovered by Berrou et al. in 1993, which comes close to the Shannon limit [13]. Due to the importance of the subject, it is worthwhile to study it from every possible angle (including DFT codes). Hopefully, this may lead to cross-fertilization and further progress.

\section{BASIC OPERATORS}

To simplify the notation, the coded signal $x$ in Fig. 1(b) will be written

$$
x=F\left[\begin{array}{l}
a \\
0
\end{array}\right]
$$

where 0 denotes a vector of $N-K$ zeros, but it is usually more convenient to preserve the conjugate symmetry inherent in the 
DFT of real data, and to take $K=2 M+1<N$, an odd integer. The set of all such $x$ is a subspace of $\mathbb{R}^{N}$ of dimension $2 M+1$ (the code subspace). The quantity $(2 M+1) / N$ can be identified with the oversampling ratio, or the code rate, for each channel.

The decoding algorithm will use two operators $B$ and $T$. The operator $B$ is a projection on the code subspace. The outputs of the two-channel coder of Fig. 1(b) satisfy $x=B x$ and $y=B y$, and

$$
B:=F\left[\begin{array}{ll}
I & 0 \\
0 & 0
\end{array}\right] F^{-1}
$$

Now note that

$$
y=F\left[\begin{array}{c}
P a \\
0
\end{array}\right]=F\left[\begin{array}{ll}
P & 0 \\
0 & 0
\end{array}\right]\left[\begin{array}{l}
a \\
0
\end{array}\right] .
$$

(For brevity, we write zero matrices of different sizes as 0 , but the size of the zero vector in (1) determines the dimensions of all of them.) Therefore, we may write $y=T x$, with

$$
T:=F\left[\begin{array}{ll}
P & 0 \\
0 & 0
\end{array}\right] F^{-1} .
$$

Also

$$
x=F\left[\begin{array}{cc}
P^{-1} & 0 \\
0 & 0
\end{array}\right] F^{-1} y=T^{H} y .
$$

Also note that

$$
T T^{H}=T^{H} T=F\left[\begin{array}{ll}
I & 0 \\
0 & 0
\end{array}\right] F^{-1}=B
$$

as well as $B T=T B=T$.

In the one-channel case, the signals would be represented using $n$ columns of $B$ (circularly shifted copies of the sampled Dirichlet kernel), whereas in the two-channel case, the $n$ columns can be choosen from $B$ and $T$. It will be seen that this new set of signals leads to much more stable representations.

\section{ONE-Channel CASE}

Consider a one-channel coder with $x \in \mathbb{R}^{N}$. Let $J$ be a nonempty subset of $\{0,1,2, \ldots, N-1\}$. Consider

$$
X:=\left\{B_{i}\right\}_{i \in J}
$$

where the $B_{i}$ are columns of $B$. Then, drawing from [3] we have the following.

1) If $X$ is a frame for the code subspace (the set of all $x$ such that $x=B x$ ), then card $J \geq 2 M+1$. If that condition holds, then there is no $x \neq 0$ such that $x=B x$ and $x_{i}=0$ for all $i \in J[10]$.

2) The frame condition

$$
\alpha\|x\|^{2} \leq \sum_{i \in J}\left|\left\langle x, B_{i}\right\rangle\right|^{2} \leq \beta\|x\|^{2}
$$

is satisfied, and the frame bounds are $\beta=\lambda_{\max }$, the largest eigenvalue of the matrix $B D B$, and $\alpha=\lambda_{\min }$, its smallest nonzero eigenvalue. The $N \times N$ matrix $D$ is defined by

$$
D_{i j}:= \begin{cases}1, & \text { if } i=j \text { and } i \in J \\ 0, & \text { otherwise. }\end{cases}
$$

When $J$ is contiguous $\alpha$ may be very close to zero, but if $J$ is more evenly distributed this may not be the case. For

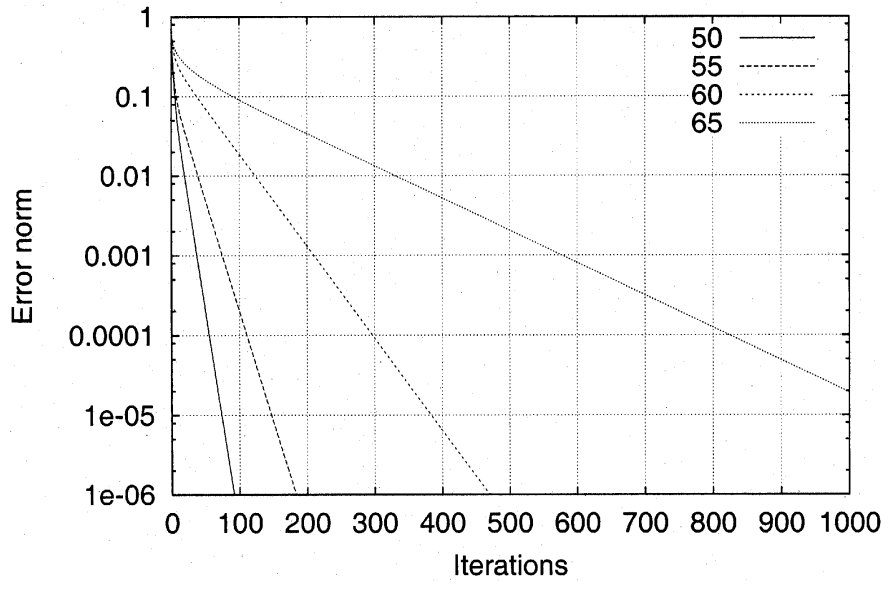

Fig. 2. Error evolution for the two-channel system, for $N=128, M=30$, and 50, 55, 60, and 65 contiguous erasures in each channel.

more on the numerical stability of the problem, see [8], [10], [14], and [15].

The basic frame algorithm is defined by

$$
f^{(n)}=f^{(n-1)}+\mu S\left(f-f^{(n-1)}\right)
$$

where $S$ is the frame operator [16], and $\mu=2 /(\alpha+\beta)$. It can be used to correct erasures since $S f$ depends only on the known samples $f_{i}, i \in J$. The convergence is geometric, but the rate

$$
\frac{\beta-\alpha}{\beta+\alpha}
$$

decreases as the ratio of the frame bounds $\beta / \alpha$ increases. The condition numbers of the matrices for noniterative decoding also increase with $\beta / \alpha$.

\section{Two-Channel CASE}

In the two-channel case, we are given $x_{0}=D_{1} x$ and $y_{0}=$ $D_{2} y$, where $D_{1}$ and $D_{2}$ are the two sampling matrices associated with the sets $J_{1}$ and $J_{2}$ that define the known data in each channel. We are in fact considering the frame $\left\{B_{i}\right\}_{i \in J_{1}} \cup$ $\left\{T_{i}\right\}_{i \in J_{2}}$, and we need a new reconstruction algorithm and an estimate for the bounds of the new frame.

To reconstruct $x$ and $y$, we use an alternating projection algorithm [17]. At the end of step $n$, there will be two approximations to $x$ and $y$, denoted by $x_{n}$ and $y_{n}$. The next approximations are computed as follows:

$$
\begin{aligned}
& x_{n+1}=x_{0}+\left(I-D_{1}\right) B x_{n} \\
& y_{n+1}=T x_{n+1} \\
& y_{n+2}=y_{0}+\left(I-D_{2}\right) B y_{n+1} \\
& x_{n+2}=T^{H} y_{n+2} .
\end{aligned}
$$

The algorithm performs remarkably well even for contiguous losses, as shown in Fig. 2, for problems well beyond the reach of the single-channel case. We will see next that the ratio of the frame bounds in the two-channel case is smaller by several orders of magnitude, a fact that explains the difference in performance. Consideration of

$$
\sum_{i \in J_{1}}\left|\left\langle x, B_{i}\right\rangle\right|^{2}+\sum_{i \in J_{2}}\left|\left\langle x, T_{i}\right\rangle\right|^{2}=\left\|D_{1} B x\right\|^{2}+\left\|D_{2} T x\right\|^{2}
$$




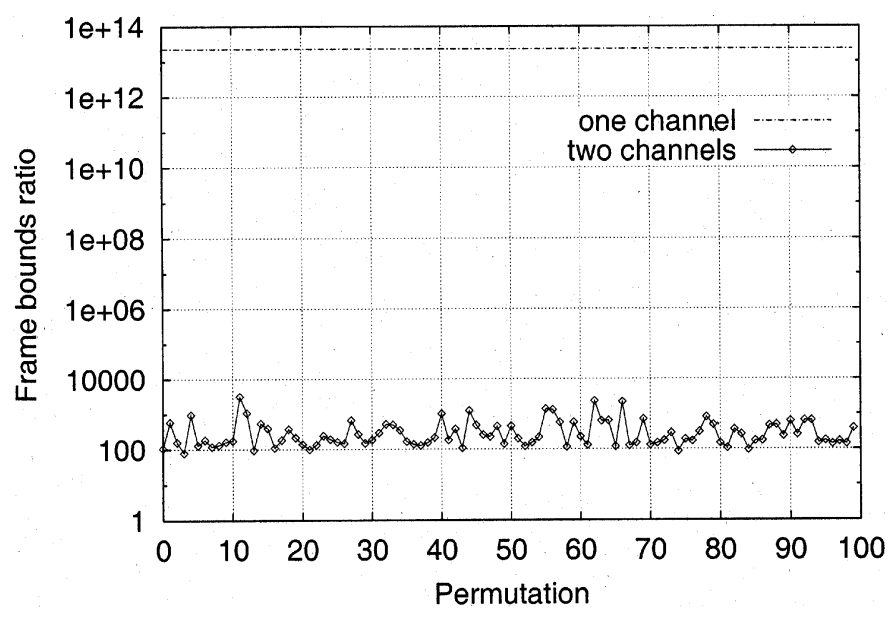

Fig. 3. Problem described in Fig. 4 was solved 100 times, each time with a new random permutation $P$. The figure shows the ratio of the frame bounds for each problem and the rate for the equivalent one-channel problem.

shows that the bounds follow from the eigenvalues of

$$
B D_{1} B+T^{H} D_{2} T \text {. }
$$

Using (2), (3), and the fact that $F$ is unitary, we see that this has the same eigenvalues as

$$
\left[\begin{array}{ll}
I & 0 \\
0 & 0
\end{array}\right] A_{1}\left[\begin{array}{ll}
I & 0 \\
0 & 0
\end{array}\right]+\left[\begin{array}{cc}
P^{T} & 0 \\
0 & 0
\end{array}\right] A_{2}\left[\begin{array}{cc}
P & 0 \\
0 & 0
\end{array}\right]
$$

where $A_{1}=F^{-1} D_{1} F$ and $A_{2}=F^{-1} D_{2} F$. Its nonzero eigenvalues are those of

$$
X+P^{T} Y P
$$

where $X$ and $Y$ are $K \times K$ submatrices of $A_{1}$ and $A_{2}$. Assume that the error patterns on both channels are equal, i.e., $Y=X$ (this includes the numerically extreme case of contiguous losses in both channels). If $v$ is an eigenvector of $X, u=P^{T} v$ will be an eigenvector of $P^{T} X P$, pertaining to the same eigenvalue. A common eigenvector $v$ of $X$ and $P^{T} X P$ pertaining to $\lambda_{\min }(X)$ would have to satisfy $v=P^{T} v$, which is impossible in general unless $P$ is a special permutation (such as the identity). Thus, in general, $\lambda_{\min }\left(X+P^{T} X P\right)$ will increase well above the minimum value $2 \lambda_{\min }(X)$, improving the numerical stability of the method and the convergence rate.

The (minor) impact of the permutation is shown in Fig. 3. In general, the best permutation $P$ depends on the patterns $D_{1}$ and $D_{2}$. Theoretically, for any given pattern one may compute the condition number of the matrices $X+P^{T} Y P$, for all $(2 M+1)$ ! possible $P$, and select those that lead to better condition numbers. However, the search is of course impractical, and Fig. 3 shows that it is also unlikely to lead to major improvements.

Fig. 4 compares the numerical stability of the one-channel and two-channel systems. Despite the oversampling ratio of $r=21 / 256$, the ratio of the frame bounds for the one-channel problem exceeds $10^{13}$. The smallest nonzero eigenvalue is already difficult to separate from the "noise floor"- the set of theoretically "zero eigenvalues." The code subspace contains signals (such as the eigenvector corresponding to the smallest

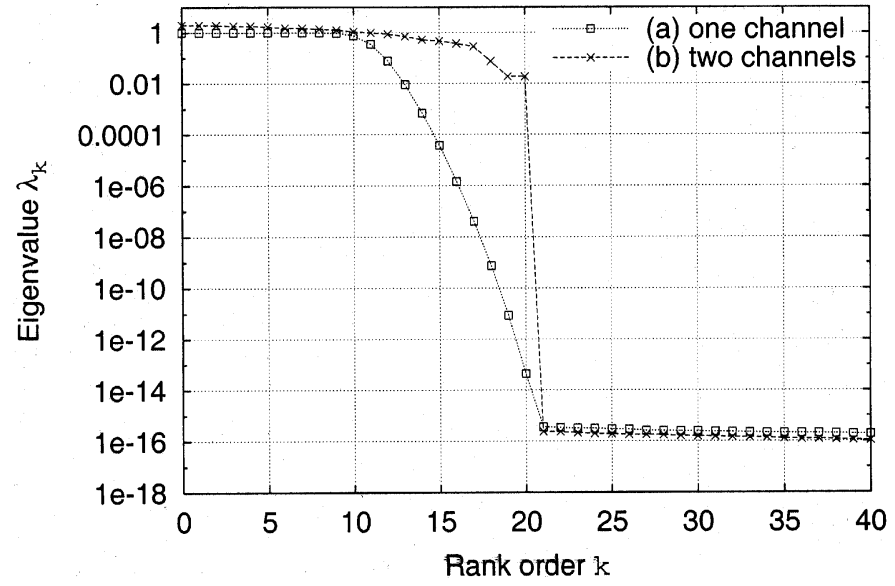

Fig. 4. (a) One-channel case. The 40 largest eigenvalues of $B D B$, for $N=256, M=10$, and 136 contiguous known samples. The ratio of the frame bounds is $>10^{13}$. (b) Two-channel case. $N=128$ samples per channel, $M=10,136$ contiguous known samples (68 per channel). The ratio of the frame bounds is $\approx 100$.

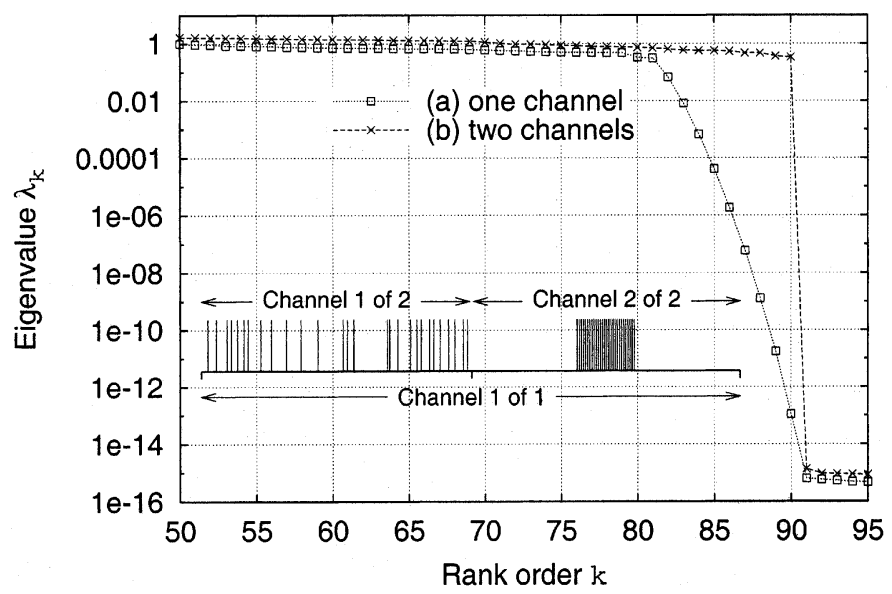

Fig. 5. (a) One-channel case. Some of the (sorted) eigenvalues of $B D B$, for $N=256, M=45$, and 200 known samples. The ratio of the frame bounds is $>10^{12}$. (b) Two-channel case. $N=128$ samples per channel, $M=45,200$ known samples ( 100 per channel). The ratio of the frame bounds is $\approx 6$. The inset shows the distribution of erasures in both cases.

nonzero eigenvalue) whose energy is almost entirely concentrated outside $J$. In the two-channel case with the same overall degree of redundancy $21 / 256$, the condition number is $\approx 100$, smaller by 11 orders of magnitude. The smallest nonzero eigenvalue is still well above the noise level of the theoretically "zero eigenvalues."

If the erasure pattern is contiguous on one-channel, but random in the other, as in the example of Fig. 5, the advantage of the two-channel method can be even greater (the reconstruction problem corresponding to the channel with random losses can be much easier numerically).

\section{CONCLUSION}

We discussed the iterative decoding of a two-channel DFT code in terms of frames and the eigenvalues of certain matrices. Its performance and numerical stability far exceeds that of single-channel codes, and works well even for bursty losses. 


\section{ACKNOWLEDGMENT}

We are indebted to J. F. Moura for bringing to our knowledge the similarity between the system depicted in Fig. 1(b) and concatenated codes with an interleaver.

\section{REFERENCES}

[1] R. E. Blahut, "Algebraic fields, signal processing, and error control," Proc. IEEE, vol. 73, pp. 874-893, May 1985.

[2] T. G. Marshall, Jr., "Coding of real-number sequences for error correction: a digital signal processing problem," IEEE J. Select. Areas Commun., vol. 2, pp. 381-391, Mar. 1984.

[3] P. J. S. G. Ferreira, "Mathematics for multimedia signal processing II-Discrete finite frames and signal reconstruction," in Signal Processing for Multimedia, J. S. Byrnes, Ed. Amsterdam, The Netherlands: IOS, 1999 , pp. 35-54

[4] J. M. N. Vieira and P. J. S. G. Ferreira, "Interpolation, spectrum analysis, error-control coding, and fault-tolerant computing," in Proc. ICASSP, vol. III, Munich, Germany, Apr. 1997, pp. 1831-1834.

[5] _ - "Noniterative reconstruction of band-limited signals and coding techniques," in Proc. 7th IEEE Digital Signal Processing Workshop, Loen, Norway, Sept. 1996, pp. 149-152.

[6] P. J. S. G. Ferreira and J. M. N. Vieira, "Locating and correcting errors in images," in Proc. ICIP, vol. I, Santa Barbara, CA, Oct. 1997, pp. 691-694.
[7] V. K. Goyal, M. Vetterli, and N. T. Thao, "Quantized overcomplete expansions in $\mathbb{R}^{N}$ : Analysis, synthesis, and algorithms," IEEE Trans. Inform. Theory, vol. 44, pp. 16-31, Jan. 1998.

[8] P. J. S. G. Ferreira, "Stability issues in error control coding in the complex field, interpolation, and frame bounds," IEEE Signal Processing Lett., vol. 7, pp. 57-59, Mar. 2000.

[9] P. Marziliano and M. Vetterli, "Reconstruction of irregularly sampled discrete-time bandlimited signals with unknown sampling locations," IEEE Trans. Signal Processing, vol. 48, pp. 3462-3471, Dec. 2000.

[10] P. J. S. G. Ferreira, "Interpolation and the discrete Papoulis-Gerchberg algorithm," IEEE Trans. Signal Processing, vol. 42, pp. 2596-2606, Oct. 1994.

[11] F. Marvasti, M. Hasan, M. Echhart, and S. Talebi, "Efficient algorithms for burst error recovery using FFT and other transform kernels," IEEE Trans. Signal Processing, vol. 47, pp. 1065-1075, Apr. 1999.

[12] G. Rath and C. Guillemot, "Performance analysis and recursive syndrome decoding of DFT codes for bursty erasure recovery," IEEE Trans. Signal Processing, 2003, to be published.

[13] S. Benedetto and E. Biglieri, Eds., Principles of Digital Transmission with Wireless Applications. New York: Kluwer Academic/Plenum, 1999.

[14] P. J. S. G. Ferreira, "The stability of a procedure for the recovery of lost samples in band-limited signals," Signal Process., vol. 40, no. 3, pp. 195-205, Dec. 1994.

[15] _ , "The eigenvalues of matrices that occur in certain interpolation problems," IEEE Trans. Signal Processing, vol. 45, pp. 2115-2120, Aug. 1997.

[16] S. G. Mallat, A Wavelet Tour of Signal Processing. San Diego, CA: Academic, 1998

[17] D. C. Youla, "Generalized image restoration by the method of alternating orthogonal projections," IEEE Trans. Circuits Syst., vol. CAS-25, pp. 694-702, Sept. 1978 